\title{
Symbolic and Cryptographic Analysis of the Secure WS-ReliableMessaging Scenario*
}

\author{
Michael Backes ${ }^{1}$, Sebastian Mödersheim² ${ }^{2}$, \\ Birgit Pfitzmann ${ }^{1}$, and Luca Viganò ${ }^{2}$ \\ 1 IBM Zurich Research Lab, Switzerland \\ 2 Information Security Group, ETH Zurich, Switzerland
}

\begin{abstract}
Web services are an important series of industry standards for adding semantics to web-based and XML-based communication, in particular among enterprises. Like the entire series, the security standards and proposals are highly modular. Combinations of several standards are put together for testing as interoperability scenarios, and these scenarios are likely to evolve into industry best practices. In the terminology of security research, the interoperability scenarios correspond to security protocols. Hence, it is desirable to analyze them for security. In this paper, we analyze the security of the new Secure WS-ReliableMessaging Scenario, the first scenario to combine security elements with elements of another quality-of-service standard. We do this both symbolically and cryptographically. The results of both analyses are positive. The discussion of actual cryptographic primitives of web services security is a novelty of independent interest in this paper.
\end{abstract}

\section{Introduction}

Web services are a series of standards that add higher-layer semantics and quality of service to web-based communication. They use XML as the basic format for all exchanged content and SOAP as the basis for message exchanges [19]. In principle, web services are independent of the underlying transport protocol; in practice, as the name suggests, typical web protocols are commonly used. An important principle of web services is modularity (see [27]). This principle was in particular applied to the design of quality-of-service features like security and message ordering. Thus, these features are addressed by a set of standards and pre-standard proposals that can, at least syntactically, be combined in a highly flexible way. It is well-known, however, that combinations of security elements have to be treated with care as many combinations may not yield the properties that one might expect. The equivalent of the classic notion of security protocols in the web-services space is interoperability profiles or scenarios. While primarily defined for interoperability testing, they are not unlikely to evolve into industry best practices for common cases. At the same

\footnotetext{
* This work was partially supported by the Zurich Information Security Center. It represents the views of the authors.
} 
time, they are at the level of concreteness where an analysis for well-known protocol security properties is possible.

In this paper, we present the first such analysis for an interoperability profile that combines features from the standards and proposals for security and another quality-of-service area, reliable messaging. It is the Secure WS-ReliableMessaging Scenario [24, which recently arose from the WS-ReliableMessaging and WS-SecureConversation Composability Interop Workshop held in April 2005 1 It is based on the WS-Security standard [36] and the recent standard proposals WS-ReliableMessaging [28] and WS-SecureConversation 32 with a few additional references to WS-Trust [33] and WS-Addressing [18]. We present two types of analysis:

1. an automated analysis based on a number of symbolic protocol analysis techniques under the assumption of perfect cryptography, and

2. an analysis closer to real cryptography based on explicit cryptographic assumptions on the underlying cryptographic algorithms used.

Both analyses refer to the properties that are already informally stated in WS-ReliableMessaging [28], where they are pointed out as desirable security properties in the context of reliable sending of messages. WS-ReliableMessaging does not address how these properties can be achieved but refers to a suitable combination with the techniques offered by the security-specific web services standards. The Secure WS-ReliableMessaging Scenario provides such a combination, and our analysis exemplifies that the properties can indeed be achieved by the techniques offered by existing web services standards.

Our first, symbolic analysis has been carried out by employing the AVISPA Tool [2,43], which is a push-button tool for the analysis of security-sensitive protocols and applications, under the assumption of perfect cryptography. The AVISPA Tool relies on a modular and expressive formal language for specifying protocols and their security properties, and integrates different back-ends that implement a variety of state-of-the-art automatic analysis techniques. For our analysis, we have employed OFMC [8] and CL-AtSe [42, which are the two more mature back-ends of the tool and which both perform protocol falsification and bounded verification by employing a number of symbolic techniques.

The Secure WS-ReliableMessaging Scenario has a structure that is far more complex than standard security protocols. Hence, an important part of modeling the protocol in a way feasible for automated analysis has been the search for a way to restrict the number of permissible interleavings of sending and receiving events without excluding attacks, i.e., every attack on the original protocol should be possible also on the simplified version. Below, we will first explain how we have built such a specification, and then illustrate the goals that we have checked in our analysis. Roughly speaking, we have shown that a client and a service mutually authenticate each other on certain messages that they exchange when executing the protocol, and that these messages remain secret.

\footnotetext{
${ }^{1}$ The title of 24] contains "scenarios" in the plural, but for our purposes the document
} defines one protocol and we thus use the singular. 
These problems give rise to an infinite search space, so that automated tools need to make restrictions on some aspects of the problem in order to analyze it. We have considered different settings by imposing bounds on the number of possible parallel protocol sessions, on the number of message sequences that can be considered in each session, and on the number of payloads per message sequence. Neither OFMC nor CL-AtSe have reported any attacks for the settings we considered, and they have thus verified the Secure WS-ReliableMessaging Scenario with respect to the modeled security properties for these settings.

Our second analysis is manual (and thus more time-consuming, less flexible to protocol additions, and more prone to human error), but more realistic with respect to the cryptographic primitives. For instance, we show that we can treat the occurring key derivation via hash functions in the standard model of cryptography as pseudo-random functions if applied to certain pairs of arguments. For the other primitives, symmetric and asymmetric encryption as well as symmetric authentication and signatures, we can use standard definitions. We also discuss how close existing theorems on justifying symbolic analyses such as our first one come to replacing a from-scratch cryptographic analysis such as our second one. Note, however, that the Secure WS-ReliableMessaging Scenario, like all other current communication security standards, does not prescribe that provably secure primitives in the cryptographic sense are used, in particular for the symmetric primitives. Thus, we cannot claim that we proved exactly the standard implementations under what became known as standard cryptographic assumptions such as the hardness of factoring. Our cryptographic analysis is modular, and some results can immediately be reused for other profiles, e.g., the analysis of the initial key exchange based mainly on WS-Trust and that of the key derivation using elements of WS-SecureConversation.

Both our analyses have positive results, i.e., they demonstrate that at the abstraction level of each analysis, the protocol is error-free. Note that our two analyses are complementary (in particular, neither of them is derived from the other), but we consider it interesting future work to investigate how to link the two kinds of analysis for web services in the style of previous proofs of soundness of Dolev-Yao models, e.g., see [1, 5, 6, 7, 20, 40.

Outline of the Paper. We start by describing the Secure WS-ReliableMessaging Scenario and the corresponding security properties in Section 2, Sections 3 and 4 contain the symbolic and the cryptographic analysis of the scenario, respectively. After reviewing further related work in Section 5 , we give concluding remarks and discuss possible future extensions of this work in Section 6 .

Due to lack of space, discussions, examples, and proofs have been shortened or omitted; details can be found in the extended version of this paper [3].

\section{The Secure WS-ReliableMessaging Scenario}

The Secure WS-ReliableMessaging Scenario is a two-party protocol initiated by a client $C$ and run together with a service $S$. It consists of three phases starting 


\section{Long-term keys:}

\begin{tabular}{|l|l|}
\hline$p k e_{X}, s k e_{X}$ & Public and secret encryption key of $X \in\{C, S\}$. \\
\hline$p k s_{X}, s k s_{X}$ & Public and secret signature key of $X \in\{C, S\}$. \\
\hline$p k s_{C A}$ & Public signature key of a certification authority $C A$. \\
\hline$C e r t_{X}$ & $\begin{array}{l}\text { Public key certificate of } X \in\{C, S\} . \text { We have } C e r t_{X}=X, p k e_{X}, p k s_{X}, \\
\operatorname{Sig}_{C A}\left(X, p k e_{X}, p k s_{X}\right), \text { where } \operatorname{Sig}_{C A}(\cdot) \text { denotes a signature computed by the } \\
\text { certification authority } C A, \text { valid with respect to } p k s_{C A} .\end{array}$ \\
\hline
\end{tabular}

\section{Cryptographic primitives:}

\begin{tabular}{|l|l|}
\hline $\operatorname{Enc}_{X}(\cdot)$ & $\begin{array}{l}\text { A public-key encryption scheme, denoting encryptions computed with public } \\
\text { key } p k e_{X} \text { for } X \in\{C, S\} .\end{array}$ \\
\hline $\operatorname{Sig}_{X}(\cdot)$ & $\begin{array}{l}\text { A digital signature scheme, denoting signatures computed with secret key } \\
s k s_{X} \text { for } X \in\{C, S\} .\end{array}$ \\
\hline $\operatorname{SymEnc}_{k}(\cdot)$ & $\begin{array}{l}\text { A symmetric encryption scheme, denoting encryptions computed with secret } \\
\text { key } k .\end{array}$ \\
\hline $\operatorname{Mac}_{k}(\cdot)$ & A message authentication code, denoting MACs computed with secret key $k$. \\
\hline $\operatorname{Hash}(\cdot)$ & A hash function, e.g., SHA-256. \\
\hline
\end{tabular}

Fig. 1. Keys and cryptographic algorithms used in the Secure WS-ReliableMessaging Scenario

\begin{tabular}{|c|c|}
\hline$I D_{1}, \ldots, I D_{9}$ & Message IDs of the individual protocol messages. \\
\hline$I D_{s k}$ & $\begin{array}{l}\text { ID of the symmetric master key } s k \text { that is established in the initial key } \\
\text { exchange phase. }\end{array}$ \\
\hline$I D_{S e q}$ & Sequence ID denoting the sequence of exchanged messages. \\
\hline$N, N^{*}$ & Nonces used to compute the master key $s k$. \\
\hline$N_{1}, N_{2}$ & $\begin{array}{l}\text { Nonces used to compute the authentication and encryption session keys } \\
s k_{1} \text { and } s k_{2} \text {. }\end{array}$ \\
\hline$m$ & Payload that should be reliably sent from $C$ to $S$. \\
\hline$n$ & Natural number denoting an acknowledged message. \\
\hline$k, k^{\prime}$ & $\begin{array}{l}\text { Symmetric keys used within a hybrid encryption in the initial key exchange } \\
\text { phase. }\end{array}$ \\
\hline$s k$ & $\begin{array}{l}\text { Symmetric master key shared between } C \text { and } S \text { after the initial key ex- } \\
\text { change phase. Derived from } N \text { and } N^{*} \text { as } s k=\operatorname{Hash}\left(N, N^{*}\right) \text {. }\end{array}$ \\
\hline$s k_{1}, s k_{2}$ & $\begin{array}{l}\text { Symmetric session keys for authentication and encryption shared between } \\
C \text { and } S \text { after the start of the message sending. Derived from } s k, N_{1} \text {, and } \\
N_{2} \text { as } s k_{i}=\operatorname{Hash}\left(N_{i}, s k\right) \text {. }\end{array}$ \\
\hline
\end{tabular}

Fig. 2. Quantities used in the Secure WS-ReliableMessaging Scenario

with a key-exchange phase, followed by the message-sending phase which uses this key, and finished by a termination phase which cancels the validity of the exchanged keys. 
We will use a straight font to denote cryptographic algorithms (Enc, Sig, etc.), a straight font with capital letters to denote protocol-specific constants (RST, RSTR, etc.), and an italic font to denote keys, identities, etc.

The key-exchange phase is based on public-key cryptography and hence requires a mechanism to authenticate the respective public keys. The profile assumes a certification authority $C A$, which has a secret key $s k s_{C A}$. Its public counterpart, $p k s_{C A}$, is known to both $C$ and $S$. The certification authority certifies the public keys of party $X \in\{C, S\}$ by signing the triple $\left(X, p k e_{X}, p k s_{X}\right)$ with its key $s k s_{C A}$, where $p k e_{X}$ and $p k s_{X}$ denote $X$ 's public encryption key and $X$ 's signature verification key, respectively. Note that $p k s_{C A}$ must have been conveyed in an authenticated manner to both $C$ and $S$, and that $p k s_{C A}$ must not give certificates with the name $X$ of an honest party to any other party.

Figures 1 and 2 summarize the notation for the keys held by both parties, the cryptographic primitives we will be using, and the quantities involved in the protocol. For interoperability, the scenario uses specific cryptographic algorithms to implement the respective primitives - RSA-1.5 for public-key encryption, RSA-SHA1 for digital signatures, AES128-CBC for symmetric encryption, and HMAC-SHA1 for message authentication codes. In the cryptographic analysis that we carry out in Section 4 , we do not fix specific algorithms but require that the used algorithms satisfy the respective security definitions under active attacks, e.g., indistinguishability under adaptive chosen-ciphertext attacks in the case of public-key encryption. Efficient schemes that satisfy these definitions exist under reasonable assumptions.

\subsection{Description of the Protocol}

Before the protocol begins, each party $X \in\{C, S\}$ has some starting information. Besides its own encryption and signature keys, the client starts with the signature verification key $p k s_{C A}$ of the certification authority $C A$, a certificate $\operatorname{Cert}_{C}$ of its own public keys, and a certificate $\operatorname{Cert}_{S}$ of the public keys of the service. The service starts only with the signature verification key $p k s_{C A}$ and with its own encryption and signature keys.

The protocol consists of nine steps, which we now briefly describe; an illustrative prose description of the individual steps based on Figures 3 [5 is given in [3]. The first two steps constitute the key-exchange phase of the protocol between the client and the service and essentially rely on the functionalities offered by WS-SecureConversation; they are depicted in Figure 3. Similarly, the last two steps cancel the validity of this key as depicted in Figure 5. Steps three to seven are depicted in Figure 4 and constitute the message-sending phase, which consists of the creation of a message sequence, the secure sending of a message $m$, and the closing of the sequence; each of these steps essentially relies on the functionalities offered by WS-ReliableMessaging.

The protocol is not simply a ping-pong protocol: after the key-exchange phase has been completed, the client is allowed to start multiple sessions of the messagesending phase in parallel and there are non-deterministic choices on the order of messages. 


\section{Composite Fields for Initial Key Exchange (Step 1-2):}

\begin{tabular}{|l|l|}
\hline body $_{1}$ & $\operatorname{SymEnc}_{k}(\mathrm{RST}, S, N)$ \\
\hline SigConf & $\operatorname{Sig}_{C}\left(I D_{1}, S, \mathrm{RST}, C\right.$, body $_{1}$, Cert $\left._{C}\right)$ \\
\hline header $_{1}$ & $\operatorname{Enc}_{S}(k), \operatorname{SymEnc}_{k}(\operatorname{SigConf})$ \\
\hline body $_{2}$ & $\operatorname{SymEnc}_{k^{\prime}}\left(\mathrm{RSTR}, I D_{\text {sk }}, S, N^{*}\right)$ \\
\hline header $_{2}$ & $\begin{array}{l}\operatorname{Enc}_{C}\left(k^{\prime}\right), \operatorname{SymEnc}_{k^{\prime}}(\text { SigConf }), \\
\operatorname{SymEnc}_{k^{\prime}}\left(\operatorname{Sig}_{S}\left(I D_{2}, C, \mathrm{RSTR}, D_{1}, \text { SigConf }, \text { body }_{2}\right)\right)\end{array}$ \\
\hline
\end{tabular}

\section{Protocol Flows (Step 1-2, from WS-SecureConversation):}
1. RequestSecurityToken:
$\mathrm{C} \stackrel{I D_{1}, S, \mathrm{RST}, C, \text { Cert }_{C}, \text { header }_{1}, \text { body }_{1}}{\longrightarrow} \mathrm{S}$
2. RequestSecurityTokenResponse: $\mathrm{C} \stackrel{I D_{2}, C, \mathrm{RSTR}, I D_{1}, \text { Cert }_{C}, \text { header }_{2}, \text { body }_{2}}{\longleftarrow} \mathrm{S}$

Fig. 3. The Key-exchange Phase, implemented via WS-SecureConversation

\section{Composite Fields for Message Sending (Step 3-7):}

\begin{tabular}{|c|c|}
\hline Session & $\left(I D_{s k}, N_{2}\right),\left(I D_{s k}, N_{1}\right)$ \\
\hline$b_{0} d y_{3}$ & $\mathrm{CS}, C, I D_{s k}$ \\
\hline header $_{3}$ & $\operatorname{SymEnc}_{s k_{2}}\left(\operatorname{Mac}_{s k_{1}}\left(I D_{3}, S, \mathrm{CS}, C, b o d y_{3}\right)\right)$ \\
\hline $\operatorname{bod}_{4}$ & CSR, ID Seq \\
\hline header $_{4}$ & $\operatorname{SymEnc}_{s k_{2}}($ SigConf $)$, SymEnc $_{s k_{2}}\left(\mathrm{Mac}_{s k_{1}}\left(I D_{4}, C, \mathrm{CSR}, I D_{3}\right.\right.$, body $\left.\left.y_{4}\right)\right)$ \\
\hline $\operatorname{bod}_{5}$ & SymEnc $_{s k_{2}}(\mathrm{PM}, m)$ \\
\hline header $_{5}$ & $\operatorname{SymEnc}_{s k_{2}}\left(\mathrm{Mac}_{s k_{1}}\left(I D_{5}, S, \mathrm{PM},\left(I D_{S e q}, n\right), b o d y_{5}\right)\right)$ \\
\hline $\operatorname{bod}_{6}$ & () \\
\hline header $_{6}$ & $\operatorname{SymEnc}_{s k_{2}}\left(\mathrm{Mac}_{s k_{1}}\left(I D_{6}, C, \mathrm{SA},\left(I D_{S e q}, n\right), \operatorname{bod} y_{6}\right)\right)$ \\
\hline $\operatorname{bod}_{7}$ & $\mathrm{TS}, I D_{S e q}$ \\
\hline header $_{7}$ & $\operatorname{SymEnc}_{s k_{2}}\left(\mathrm{Mac}_{s k_{1}}\left(I D_{7}, S, \mathrm{TS},\left(\mathrm{TS}, I D_{S e q}\right), \operatorname{bod} y_{7}\right)\right)$ \\
\hline
\end{tabular}

\section{Message Sending (Step 3-7, from WS-ReliableMessaging):}
3. CreateSequence:
$\mathrm{C} \longrightarrow \mathrm{ID} D_{3}, \mathrm{~S}, \mathrm{CS}$, Session, header 3, body $_{3} \longrightarrow \mathrm{S}$
4. CreateSequenceResponse:
$\mathrm{C} \longleftarrow \mathrm{ID}_{4}, C, \mathrm{CSR}$, Session, header 4, body $_{4}$ $I D_{5}, S, \mathrm{PM},\left(I D_{S e q}, n\right), I D_{s k}$, Session,
5. PayloadMessage: $\mathrm{C}$

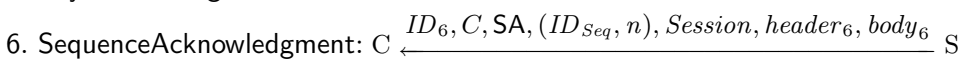
7. TerminateSequence:
$\mathrm{C} \stackrel{I D_{7}, S, \mathrm{TS}, I D_{s k}, \text { Session, header }}{7}$, body $_{7} \longrightarrow \mathrm{S}$

Fig. 4. The Message-sending Phase, implemented via WS-ReliableMessaging 


\section{Composite Fields for Session Closure (Step 8-9):}

\begin{tabular}{|c|c|}
\hline Session & $\left(I D_{s k}, N_{2}\right),\left(I D_{s k}, N_{1}\right)$ \\
\hline body 8 & $\mathrm{CST}, I D_{s k}$ \\
\hline header $_{8}$ & $\operatorname{SymEnc}_{s k_{2}}\left(\mathrm{Mac}_{s k_{1}}\left(I D_{8}, S, \mathrm{CST}, C, b o d y_{8}\right)\right.$ \\
\hline$b_{0} d y_{9}$ & CSTR \\
\hline header $_{9}$ & $\begin{array}{l}\operatorname{SymEnc}_{s k_{2}}(\operatorname{SigConf}), \\
\operatorname{SymEnc}_{s k_{2}}\left(\operatorname{Mac}_{s k_{1}}\left(I D_{9}, C, \mathrm{CSTR},\left(I D_{s k}, N_{1}\right), \operatorname{body} y_{9}\right)\right.\end{array}$ \\
\hline
\end{tabular}

\section{Protocol Flows (Step 8-9, from WS-SecureConversation):}
8. CancelSecurityToken:
$\mathrm{C} \stackrel{I D_{8}, S, \mathrm{CST}, C, I D_{s k}, \text { Session, header } 8, \text { body }_{8}}{\longrightarrow} \mathrm{S}$
9. CancelSecurityTokenResp: C
ID ${ }_{9}, C$, CSTR, Session, header 9, body $_{9}$ S

Fig. 5. The Termination Phase, implemented via WS-SecureConversation

The necessary tests on the received messages follow the usual convention as described in 35], e.g., an honest receiver of a message checks that the decrypted plaintexts are of the correct format, that respective parts of the plaintext match corresponding parts sent unencrypted in the same message, and that the sender and receiver fields contain the expected values. We do not always mention this explicitly in the following.

Possible Protocol Extensions. We moreover sketch a possible extension of the interoperability scenario to reflect additional capabilities of the client and the service offered by the WS-ReliableMessaging standard. The standard

\section{Composite Fields for Protocol Extension:}

\begin{tabular}{|c|c|}
\hline$I D_{5.1}, I D_{5.1^{*}}$ & Message IDs of the additional protocol messages. \\
\hline $\operatorname{bod}_{5.1}$ & () \\
\hline header $_{5.1}$ & $\operatorname{SymEnc}_{s k_{2}}\left(\mathrm{Mac}_{s k_{1}}\left(I D_{5.1}, C, \mathrm{NAck},\left(I D_{S e q}, n\right), \operatorname{bod} y_{5.1}\right)\right)$ \\
\hline $\operatorname{body}_{5.1^{*}}$ & () \\
\hline header $_{5.1^{*}}$ & $\operatorname{SymEnc}_{s k_{2}}\left(\operatorname{Mac}_{s k_{1}}\left(I D_{5.1^{*}}, C, \mathrm{AR},\left(I D_{S e q}, n\right), \operatorname{bod} y_{5.1^{*}}\right)\right)$ \\
\hline
\end{tabular}

\section{Resend and Ack Inquiries (Between Step 5 and 6, from WS- ReliableMessaging):}

5.1. NotAcknowledged: C $\stackrel{I D_{5.1}, C \text {, NAck, }\left(I D_{S e q}, n\right), \text { Session, header }{ }_{5.1}, \text { body }_{5.1}}{\mathrm{~S}}$

5.1*. AckRequested: $\quad \mathrm{C} \stackrel{I D_{5.1^{*}}, C, \mathrm{AR},\left(I D_{\text {Seq }}, n\right), \text { Session, header }{ }_{5.1^{*}}, \text { body }_{5.1^{*}}}{\longrightarrow} \mathrm{S}$

Fig. 6. Extension of the Secure WS-ReliableMessaging Scenario with Resend Inquiries 
additionally allows a client to request an unreceived acknowledgment of a previously sent message, and it allows a service to ask the client to re-send a message if it has not been received yet. This yields two additional steps which are depicted in Figure6.

\subsection{Security Properties}

We consider a range of reasonable security requirements for the parties involved; some of the requirements are explicitly mandated by the standards, others are optional and hold only under stronger assumptions on the underlying cryptographic primitives. The following security properties are explicitly pointed out in WS-ReliableMessaging:

- No Message Alteration: Payloads contained in the 5. PayloadMessage in a session between an honest client and an honest service cannot be altered by an adversary.

- No Message Disclosure: Payloads contained in the 5. PayloadMessage in a session between an honest client and an honest service remain secret from the adversary.

- Key Integrity and Confidentiality: If an honest client and an honest service established a shared key $s k$ after the first two steps of the protocol, both parties obtained the same key. Moreover, this key is secret from the adversary.

- Authentication: If an honest service accepts a payload $m$ presumably from an honest client, then this honest client indeed sent this payload in the same session.

Accountability is also mentioned in WS-ReliableMessaging as one of the properties desirable in certain scenarios. As this scenario uses symmetric cryptography for the message authentication, accountability in the sense of non-repudiation is clearly not a goal of this scenario. The potential real-life accountability of this scenario is formally captured on the protocol level by the message integrity property and otherwise given by non-protocol factors. We refer to [3] for additional useful properties that are not explicitly required by the standard as well as for a refinement of the aforementioned properties tailored to the Secure WS-ReliableMessaging Scenario.

\section{Symbolic Security Analysis}

The AVISPA Tool. We have carried out a symbolic analysis of the Secure WS-ReliableMessaging Scenario by employing the AVISPA Tool [2, 43], which is a push-button tool for the automated validation, under the assumption of perfect cryptography and Dolev-Yao adversary [25], of industrial-scale Internet security-sensitive protocols and applications. A user interacts with the AVISPA Tool by specifying a security problem (a protocol paired with a security property that it is expected to achieve) in the High-Level Protocol Specification Language 
HLPSL 21, which is an expressive, modular, role-based, formal language that allows for the specification of control-flow patterns, data structures, alternative adversary models, complex security properties, as well as different cryptographic operators and their algebraic properties. The AVISPA Tool automatically translates a user-defined security problem into an equivalent description of an infinitestate transition system that is then input to the back-ends of the AVISPA Tool. The back-ends search the transition system for states that represent attacks on the intended properties of the protocol.

The current version [2, 43] of the tool integrates four back-ends that implement a variety of state-of-the-art automatic analysis techniques, ranging from protocol falsification (by finding an attack on the input protocol) to abstraction-based verification methods for infinite numbers of sessions. The back-ends are: the On-the-fly Model-Checker OFMC, the Constraint-Logic-based Attack Searcher $C L$-AtSe, the SAT-based Model-Checker SATMC, and the TA4SP verifier, which analyzes protocols by implementing tree automata based on automatic approximations. All the back-ends of the tool analyze protocols by considering the standard Dolev-Yao model of an active adversary that controls the network but cannot break cryptography; in particular, the adversary can intercept messages and analyze them if it possesses the respective keys for decryption, and it can generate messages from his knowledge and send them under any party's name. Upon termination, the AVISPA Tool outputs that the protocol was verified with respect to the specified security problem, that an attack was found, or that the available resources were exhausted.

For our analysis of the Secure WS-ReliableMessaging Scenario, we have employed OFMC [8] and CL-AtSe [42, which are the two more mature back-ends of the tool, with better scope and performance. OFMC and CL-AtSe both perform protocol falsification and bounded verification by employing a number of symbolic techniques. Some of these techniques are back-end specific, while other ones are common to the two back-ends, such as the lazy intruder technique to symbolically represent all the possible messages that the Dolev-Yao adversary can generate. These techniques enable both OFMC and CL-AtSe to handle protocols with complex message terms and in particular to model the Secure WS-ReliableMessaging Scenario in its full complexity, without having to simplify the messages that are exchanged 2

The Model. The back-ends of the AVISPA Tool have successfully validated (or found a number of new attacks on) security protocols such as those in the Clark/Jacob library [22], as well as Kerberos, IKE, SET, and other protocols proposed by standardization organizations such as the IETF, ITU, W3C, Oasis, IEEE, 3GPP, and OMA. Similar analyses have been carried out by other (semi-)automated tools such as [9, 16, 17, 26, 41].

\footnotetext{
${ }^{2}$ The complexity of the Scenario prevents the usage of the current versions of SATMC and TA4SP. We hope to soon be able to report on the analysis with these back-ends as well; in particular, if analysis with TA4SP succeeded, then that would prove that the protocol is safe for secrecy goals for any number of sessions.
} 
The Secure WS-ReliableMessaging Scenario has a structure that is far more complex than that of standard security protocols. Nonetheless, thanks to its expressiveness, HLPSL allows us to completely model the protocol, i.e., to provide a formal specification of the complex interactions between the two honest parties, which we can model as two separate client and service programs that communicate over an insecure network controlled by a Dolev-Yao adversary. However, such a model is too complex for automated analysis as even for a limited number of sessions, the set of permissible interleavings of sending and receiving events is enormous. For instance, the messages sent by the client may arrive in any order at the service. Additionally, both the client and the service can send "administrative" messages, i.e., acknowledge messages, request the retransmission of messages, or request the acknowledgment of messages. An important part of modeling the protocol in a way feasible for automated analysis has thus been the search for a way to restrict the number of interleavings without excluding attacks, i.e., such that any attack on the original protocol is possible also on the simplified version.

We have performed a step-by-step simplification of the client and service programs, whereby we have showed that these simplifications do not exclude any attacks 3 As we lack space to give the HLPSL specification here due to its complexity and the amount of explanation that would be necessary, we only sketch the main ideas behind our HLPSL specification. In particular, we briefly illustrate the simplifications we have carried out for the client program; the ones for the service program are similar, and more details can be found in [3, together with a formal justification of the fact that these simplifications of the HLPSL specification do not exclude any attacks.

In order to simplify the client, note, firstly, that it is not a restriction if the client sends in one transition all the messages that it wishes to transmit via the 5. PayloadMessage step as soon as it has received the 4. CreateSequenceResponse message. Secondly, the client can neglect any requests of step 5.1. NotAcknowledged from the service to retransmit messages, since the Dolev-Yao adversary has seen all messages and can thus replay them to the service if this is necessary for an attack. Hence, we can consider a simplified client program that, having sent all its payload messages, simply waits for acknowledgment messages (6. SequenceAcknowledgment) or, after timing out, requests acknowledgment from the service (5.1* . AckRequested). Thirdly, since the Dolev-Yao adversary can intercept all responses from the service, it might deliberately make the client produce acknowledge request messages. Hence we can assume that the adversary can obtain acknowledge request messages of step 5.1*. AckRequested for every payload message. No attacks are therefore excluded if the client program sends with every 5. PayloadMessage also an 5.1*. AckRequested message.

\footnotetext{
${ }^{3}$ The simplified (restricted) version of the protocol that we obtain in this way is only useful for the formal analysis, not for the practical deployment of the protocol: for instance, since a Dolev-Yao adversary can replay old messages arbitrarily if this is necessary to mount an attack, we can restrict the model to client programs that never retransmit old messages.
} 
These simplifications yield a client program that behaves as follows in every message sending phase: it sends all payload messages together with the corresponding requests for acknowledgment in one step, then waits until all messages are acknowledged, and finally sends a 7 . TerminateSequence message.

Goals. Let us define the security-relevant messages of the Secure WSReliableMessaging Scenario to be the key-material $\left(s k, s k_{1}\right.$, and $\left.s k_{2}\right)$ and all payloads transmitted with a 5. PayloadMessage. For our symbolic analysis, we have specified a number of secrecy and authentication goals (giving rise to different HLPSL security problems for the Scenario):

- secrecy of all security-relevant messages, and

- mutual authentication between client and service on all security-relevant messages.

We model these goals by labeling several transitions in the HLPSL specification with special events that express the meaning of the transition with respect to the goals of the protocol. First, whenever a client $\mathrm{c}$ that believes to talk with service $\mathbf{s}$ creates a security-relevant message $\mathrm{m}$, then it generates a secret event secret $(m,\{c, s\})$ expressing that $m$ must remain secret between the parties in the specified set, in this case $\mathrm{c}$ and $\mathbf{s}$. This allows us to define a violation of secrecy by a state of the transition system in which the adversary knows a message $\mathrm{m}$ for which a secrecy event has occurred with a set of parties to which the adversary does not belong. Second, we define violations of authentication by labeling the transitions with witness and request events. Whenever a party a that believes to talk with another party b first "handles" some security-relevant message $m$ (i.e., either creates it or receives it for the first time), then it generates an event witness $(a, b, i d, m)$ where id is an identifier that uniquely determines the purpose of the message in the protocol. This witness event expresses that a uses message $\mathrm{m}$ for communication with $\mathrm{b}$ and for purpose $i d$. The service $\mathrm{s}$ generates an event request $(\mathrm{s}, \mathrm{c}, \mathrm{id}, \mathrm{m})$ when it receives a payload $\mathrm{m}$ (supposedly) from the client c with index id. Similarly, if the client c receives the acknowledgement for the id-th payload (supposedly) from the service $\mathbf{s}$, and if $\mathrm{c}$ has previously sent $\mathrm{m}$ as the id-th payload, then $\mathrm{c}$ generates the event request $(\mathrm{c}, \mathrm{s}, \mathrm{id}, \mathrm{m})$. Similar request events are generated for the authentication on the key-material. (Intuitively, request events express that a party begins to rely on the agreement with another party on the specified value.)

A violation of authentication is then defined as any of the two following situations. First, weak authentication is violated whenever there is a request $(b, a, i d, m)$ but no matching witness event witness $(a, b, i d, m)$, i.e., a party $\mathrm{b}$ believes a message $\mathrm{m}$ to come from $\mathrm{a}$, but a has never sent $\mathrm{m}$, at least not for this purpose. Second, strong authentication is violated whenever weak authentication is, or whenever a request event occurs more frequently than the corresponding witness event (i.e., by a kind of replay, the adversary made party $\mathrm{b}$ accept a message more often than it was actually said by a). Note that these goals are equivalent to Lowe's [39] notions of non-injective and injective agreement, respectively. 
The security problems that we obtain by modeling these goals cover the main security properties stated for the Secure WS-ReliableMessaging Scenario in Section 2.2 as follows:

- secrecy of all security-relevant messages covers no message disclosure and key confidentiality,

- mutual authentication between client and service on all security-relevant messages covers no message alteration, key integrity, and authentication.

Bounds of the Analysis. The security problems associated with the Secure WS-ReliableMessaging Scenario give rise to an infinite search space, so that, in order to analyze this space, automated tools need to make some restrictions, i.e., to impose some bounds to consider relevant protocol execution and analysis settings. In the following, we will describe the restrictions that we imposed in our analysis with OFMC and CL-AtSe.

In general, there is no bound on the number of parties and sessions of the protocol that can be executed in parallel. While one can bound the number of parties, by the argumentations of [23] or by the symbolic sessions technique of OFMC [8, the problem of an unbounded number of sessions cannot be solved in general since it gives rise to undecidability. Moreover, there are two similar problems of unboundedness in the protocol: there is no bound on the number of payload messages to be exchanged or on the number of new message sequences that can be started, i.e., the protocol contains unbounded loops. All these problems give rise to an unbounded number of steps of honest parties, while both OFMC and CL-AtSe currently require analysis settings with bounded numbers of steps of honest parties.

In general, there is also no bound on the complexity of messages that the adversary can generate. However, as we remarked above, both OFMC and CL-AtSe implement the lazy intruder technique, which uses a symbolic representation to avoid explicitly enumerating the possible messages that the Dolev-Yao adversary can generate, and which allows for an analysis without restricting this parameter of the problem.

We have therefore analyzed the protocol with OFMC and CL-AtSe under the following execution/analysis settings: there are at most three parallel protocol sessions, the client can start at most two message-sending sequences per protocol session, and there are at most three payload messages per message sequence. Neither OFMC nor CL-AtSe have reported any attacks on the protocol for these analysis settings. In particular, for three parallel sessions, both OFMC and CLAtSe verified the protocol within three hours (while the verification of smaller settings required between few seconds to a minute).

\section{Cryptographic Security Analysis}

In this section, we complement the symbolic analysis of the security properties of the WS-ReliableMessaging Scenario from Section 3 by a cryptographic analysis. Thus we now analyze the security of the scenario in a cryptographic setting 
where the cryptographic primitives and the perfect cryptography assumption are replaced with actual cryptographic algorithms and the corresponding security notions that reason about probabilistic polynomial-time attackers. It is known that, even if the symbolic analysis is careful in distinguishing primitives like symmetric encryption and authentication, as both the analyzed scenario and the analysis in Section 3 do, and even if one assumes that an implementation is made with primitives secure according to the strictest usual cryptographic definitions, the results of such a symbolic analysis may not carry over to the real implementation. The most prominent example is that it cannot be avoided in general that the length of encrypted payload data, such as the values $m$ in the PayloadMessage, leaks. Other problems that may occur in general scenarios are due to the probabilism of secure public-key encryption, key-stealing attacks, and manipulations of symmetric encryptions unless authenticated encryption [11,10 is used in the implementation [5,4. Consequently, in a Dolev-Yao-style cryptographic library designed to be implemented based on arbitrary cryptographically secure primitives and to be usable in a secure way within arbitrary protocols with arbitrary security properties, both the abstraction and the realization must have certain idiosyncrasies. Hence, while it might be interesting to augment a tool like the AVISPA Tool by the idiosyncrasies of the Dolev-Yao style model of [5, 6, 4, and while implementing the primitives of WS-Security with the extended realizations from those papers (e.g., some additional tagging and randomization) might realize the goal of web service security to offer completely composable primitives also in a semantic sense, neither has been done yet. Other work on bridging the gap between symbolic and cryptographic security concentrated more on keeping very close to standard symbolic and real versions at the cost of generality. However, at present none of them covers the protocol class of Secure WS-ReliableMessaging, nor the security properties required. The seminal work [1] treats passive attacks only. Active attacks have been considered in this context in $40,38,20$. First, however, each of these papers treats only one cryptographic primitive, asymmetric encryption in [40, 20] and symmetric encryption in 38. Secondly, 40, only treats integrity properties, while 38, only treats the secrecy of fixed, protocol-internal messages and [20] only treats the secrecy of nonces, i.e., random values chosen within the protocol and not usable for operations (such as encrypting) in that protocol. It may be interesting future work to extend such results on restricted usage of cryptographic libraries to the typical usage in WS-Security protocols. Our following considerations can be seen as a step in this direction.

Given these shortcomings of the current methods for deducing the security in the cryptographic setting from a symbolic proof, we do not try to do that, but base our proof directly on existing cryptographic work that explored the security of encryption, signatures, and MACs when combined in specific ways. In the following, we assume that the public-key encryption system Enc be secure against adaptive-chosen ciphertext attacks (short IND-CCA2-secure), that the symmetric encryption scheme be secure under adaptive chosen-plaintext attacks (short IND-CPA-secure), and that the signature scheme Sig and the message 
authentication scheme Mac be secure against adaptive chosen-message attacks (short IND-CMA-secure). These are the commonly accepted security definitions of these primitives under active attacks so that we omit their rigorous definition. Primitives secure in this sense exist under reasonable assumptions.

Furthermore, we have to require that the hash function Hash used to compute the secret key $s k$ based on two secret nonces does not degenerate the randomness induced by the nonces. This would be clear if we worked in the random oracle model; however, the specific setting of the scenario allows us to work in the standard model with a sufficient condition being that Hash, when applied to pairs, is a pseudo-random function in its first argument.

We obtain the following theorem (proven in [3]), in which we assume that the Secure WS-ReliableMessaging Scenario is run as a stand-alone protocol. This is not necessarily realistic for a web-services implementation; then our approach may have to take policies into account as in [14].

Theorem 1. (Cryptographic Security of Secure WS-Reliable Messaging Scenario) If Enc is IND-CCA2-secure, if Sig is IND-CMA-secure, if SymEnc is INDCPA-secure, and if Hash, when applied to pairs, is a pseudo-random function in its first argument, then key integrity and key confidentiality are cryptographically fulfilled for the scenario, i.e., if the protocol is run with a probabilistic polynomial-time adversary, the keys are authentic with overwhelming probability, and the keys are indistinguishable from fresh random keys given the view of the adversary. If additionally Mac is IND-CMA-secure, then message integrity and no message disclosure are cryptographically fulfilled.

\section{Further Related Literature}

Work is currently underway on scaling-up formal analysis methods and tools to web services security protocols, e.g., 12, 13, 14, 15, 29, although none of these works performs a cryptographic analysis of the protocols. In particular, the TulaFale tool 15. compiles descriptions of XML/SOAP-based security protocols and properties into the applied pi calculus and then employs the ProVerif tool [16. We considered employing also TulaFale for the automatic symbolic analysis of Secure WS-ReliableMessaging, but its input language would first need to be extended to express all the constructs of the profile, and we thus leave this analysis and the comparison with our own symbolic analysis as future work. Recent work has also considered the automated analysis of XML-based web services: 37. presents a formal analysis of an encoding of the original XML messages into standard security protocol notation, showing that this encoding is without loss of attacks. Based on this encoding, the Casper/FDR tool can then check security properties for an unbounded number of sessions thanks to the employed data independence technique (which is similar to the abstraction techniques in TA4SP). The considered protocol, however, is simpler than the Secure WS-ReliableMessaging Scenario (e.g., no open-ended exchange of payload messages) and its analysis with Casper/FDR required simplifications of the 
message terms. It is thus not clear if the method of [37 could also work on complex protocols such as the one considered in this paper.

Another type of analysis of a web services security protocol is that of an interoperability profile of WS-Federation in [31]. The analyzed profile [34] is a passive requestor profile, i.e., the user is represented only by a browser. The emphasis therefore lies on treating a browser in a protocol security proof. The analysis is by hand, and as only signatures and secure channels occur as cryptographic primitives, there is not much discussion of detailed properties of the cryptographic primitives in web services.

\section{Conclusion and Outlook}

We have given a symbolic and a cryptographic analysis of the security of the new Secure WS-ReliableMessaging Scenario, which constitutes the first web services scenario to combine security elements with elements of another quality-of-service standard. The results of both analyses are positive, i.e., they are proofs as far as the techniques faithfully represent the standards; these restrictions concern the cryptographic primitives and, in the symbolic case, the analysis settings. Our symbolic analysis is a further step in the use of formal proof tools for the validation of security protocols and web services under the perfect cryptography assumption. Our cryptographic analysis constitutes an important first step to reason about the security of web services in the more realistic setting where the perfect cryptography assumption is replaced by the complexity-theoretic definitions of cryptography. Some of the cryptographic results are of more general applicability in web services security than for the specific settings analyzed here.

As future work on the symbolic side, we have begun considering additional symbolic analysis settings, as well as employing abstraction techniques for carrying out unbounded verification. To this end, it would be particularly interesting not only to employ AVISPA's TA4SP, but also to investigate the relationships and possible complementarity of our analysis with an analysis carried out by TulaFale/ProVerif, especially since the model checkers that we used implement different techniques than those of ProVerif (which combines symbolic representations based on first-order logic and abstractions). Moreover, it would be of great help to be able to exploit the automatic compilation provided by TulaFale and we will thus investigate how to do so for the AVISPA Tool. We believe that the work of [30] will be helpful here, as it provides a preliminary translation procedure from protocol descriptions in HLPSL to descriptions in the applied pi calculus, which thus allows one to apply the ProVerif tool to some existing HLPSL protocol specifications.

On the cryptographic side, it would be interesting to see in which respect one can weaken the security requirements imposed on the cryptographic primitives without invalidating the security properties. Furthermore, we intend to apply our techniques to other profiles and scenarios and possibly even to a policy-based analysis similar to 14 on the symbolic side. 


\section{References}

1. M. Abadi and P. Rogaway. Reconciling two views of cryptography: The computational soundness of formal encryption. In Proc. 1st IFIP TCS, LNCS 1872, pp. 3-22. Springer, 2000.

2. A. Armando, D. Basin, Y. Boichut, Y. Chevalier, L. Compagna, J. Cuellar, P. Hankes Drielsma, P.-C. Heám, O. Kouchnarenko, J. Mantovani, S. Mödersheim, D. von Oheimb, M. Rusinowitch, J. Santiago, M. Turuani, L. Viganò, and L. Vigneron. The AVISPA Tool for the Automated Validation of Internet Security Protocols and Applications. In Proc. CAV'2005, LNCS 3576, pp. 281-285. Springer, 2005.

3. M. Backes, S. Mödersheim, B. Pfitzmann, and L. Viganò. Symbolic and Cryptographic Analysis of the Secure WS-ReliableMessaging Scenario (Extended Version). Technical Report 502, Department of Computer Science, ETH Zurich, 2006. Available at www.infsec.ethz.ch

4. M. Backes and B. Pfitzmann. Symmetric encryption in a simulatable Dolev-Yao style cryptographic library. In Proc. 17th IEEE CSFW, 2004.

5. M. Backes, B. Pfitzmann, and M. Waidner. A composable cryptographic library with nested operations (extended abstract). In Proc. 10th ACM CCS, pp. 220 230, 2003. Full version in IACR Cryptology ePrint Archive 2003/015, Jan. 2003, http://eprint.iacr.org/,

6. M. Backes, B. Pfitzmann, and M. Waidner. Symmetric authentication within a simulatable cryptographic library. In Proc. 8th ESORICS, LNCS 2808, pp. 271290. Springer, 2003.

7. M. Backes, B. Pfitzmann, and M. Waidner. A general composition theorem for secure reactive systems. In Proc. 1st TCC, LNCS 2951, pp. 336-354. Springer, 2004 .

8. D. Basin, S. Mödersheim, and L. Viganò. OFMC: A Symbolic Model-Checker for Security Protocols. International Journal of Information Security, 4(3):181-208, 2005 .

9. G. Bella, F. Massacci, and L. C. Paulson. Verifying the SET Purchase Protocols. Journal of Automated Reasoning, to appear.

10. M. Bellare and C. Namprempre. Authenticated encryption: Relations among notions and analysis of the generic composition paradigm. In Proc. ASIACRYPT 2000, LNCS 1976, pp. 531-545. Springer, 2000.

11. M. Bellare and P. Rogaway. Encode-then-encipher encryption: How to exploit nonces or redundancy in plaintexts for efficient constructions. In Proc. ASIACRYPT 2000, LNCS 1976, pp. 317-330. Springer, 2000.

12. K. Bhargavan, R. Corin, C. Fournet, and A. Gordon. Secure sessions for web services. In Proc. ACM Workshop on Secure Web Services (SWS), 2004.

13. K. Bhargavan, C. Fournet, and A. Gordon. A semantics for web service authentication. In Proc. 31st POPL, pp. 198-209. ACM Press, 2004.

14. K. Bhargavan, C. Fournet, and A. Gordon. Verifying policy-based security for web services. In Proc. 11th ACM CCS, pp. 268-277, 2004.

15. K. Bhargavan, C. Fournet, A. Gordon, and R. Pucella. TulaFale: A security tool for web servics. In Proc. 2nd FMCO, LNCS 3188, pp. 197-222. Springer, 2004.

16. B. Blanchet. An efficient cryptographic protocol verifier based on Prolog rules. In Proc. 14th IEEE CSFW, pp. 82-96, 2001.

17. C. Bodei, M. Buchholtz, P. Degano, F. Nielson, and H. Riis Nielson. Static validation of security protocols. Journal of Computer Security, 13(3):347-390, 2005.

18. D. Box, F. Curbera et al. Web Services Addressing (WS-Addressing), Aug. 2004. 
19. D. Box, D. Ehnebuske, G. Kakivaya, A. Layman, N. Mendelsohn, H. F. Nielsen, S. Thatte, and D. Winer. Simple object access protocol (SOAP) 1.1, May 2000.

20. R. Canetti and J. Herzog. Universally composable symbolic analysis of cryptographic protocols (the case of encryption-based mutual authentication and key exchange). Cryptology ePrint Archive, Report 2004/334, 2004.

21. Y. Chevalier, L. Compagna, J. Cuellar, P. Hankes Drielsma, J. Mantovani, S. Mödersheim, and L. Vigneron. A High Level Protocol Specification Language for Industrial Security-Sensitive Protocols. In Proc. Workshop on Specification and Automated Processing of Security Requirements (SAPS'04), pp. 193-205. Austrian Computer Society, 2004.

22. J. Clark and J. Jacob. A Survey of Authentication Protocol Literature: Version 1.0, 17. Nov. 1997.

23. H. Comon-Lundh and V. Cortier. Security properties: two agents are sufficient. In Proc. 12th ESOP, LNCS 2618, pp. 99-113. Springer, 2003.

24. D. Davis, C. Ferris, V. Gajjala, K. Gavrylyuk, M. Gudgin, C. Kaler, D. Langworthy, M. Moroney, A. Nadalin, J. Roots, T. Storey, T. Vishwanath, and D. Walter. Secure WS-ReliableMessaging scenarios, Apr. 2005. ftp://www6. software.ibm.com/software/developer/library/ws-rmseconscenario.doc.

25. D. Dolev and A. C. Yao. On the security of public key protocols. IEEE Transactions on Information Theory, 29(2):198-208, 1983.

26. B. Donovan, P. Norris, and G. Lowe. Analyzing a library of security protocols using Casper and FDR. In Proc. Workshop on Formal Methods and Security Protocols (FMSP'99), 1999.

27. D. F. Ferguson, T. Storey, B. Lovering, and J. Shewchuk. Secure, reliable, transacted Web Services - architecture and composition, Oct. 2003. Available at http://www-106.ibm.com/developerworks/webservices/library/ ws-securtrans/.

28. C. Ferris, D. Langworthy et al. Web Services Reliable Messaging Protocol (WSReliableMessaging), Feb. 2005.

29. A. Gordon and R. Pucella. Validating a web service security abstraction by typing. In Proc. 1st ACM Workshop on XML Security, pp. 18-29, 2002.

30. A. Gotsman, F. Massacci, and M. Pistore. Towards an Independent Semantics and Verification Technology for the HLPSL Specification Language. Electronic Notes in Theoretical Computer Science 135(1):59-77, 2005.

31. T. Groß, B. Pfitzmann, and A.-R. Sadeghi. Proving a WS-Federation Passive Requestor Profile with a Browser Model. In Proc. ACM Workshop on Secure Web Services (SWS), pp. 54-64. ACM Press, 2005.

32. M. Gudgin, A. Nadalin et al. Web Services Secure Conversation Language (WSSecureConversation), Feb. 2005.

33. M. Gudgin, A. Nadalin et al. Web Services Trust Language (WS-Trust), Feb. 2005.

34. M. Hur, R. D. Johnson, A. Medvinsky, Y. Rouskov, J. Spellman, S. Weeden, and A. Nadalin. Passive Requestor Federation Interop Scenario, Version 0.4, Feb. 2004. ftp://www6. software.ibm.com/software/developer/library/ws-fpscenario2. doc.

35. F. Jacquemard, M. Rusinowitch, and L. Vigneron. Compiling and verifying security protocols. In Proc. LPAR 2000, LNCS 1955, pp. 131-160. Springer, 2000.

36. C. Kaler et al. Web Services Security (WS-Security), version 1.0, Apr. 2002.

37. E. Kleiner and A. Roscoe. On the relationship of traditional and Web Services Security protocols. In Proceedings of the XXI Mathematical Foundations of Programming Semantics (MFPS'05). Electronic Notes in Theoretical Computer Science, to appear. 
38. P. Laud. Symmetric encryption in automatic analyses for confidentiality against active adversaries. In Proc. 25th IEEE Symposium on Security \& Privacy, pp. 71-85, 2004.

39. G. Lowe. A hierarchy of authentication specifications. In Proc. 10th IEEE CSFW, pp. 31-43, 1997.

40. D. Micciancio and B. Warinschi. Soundness of formal encryption in the presence of active adversaries. In Proc. 1st TCC, LNCS 2951, pp. 133-151. Springer, 2004.

41. D. Song, S. Berezin, and A. Perrig. Athena: a novel approach to efficient automatic security protocol analysis. Journal of Computer Security, 9:47-74, 2001.

42. M. Turuani. Sécurité des Protocoles Cryptographiques: Décidabilité et Complexité. Phd, Université Henri Poincaré, Nancy, December 2003.

43. L. Viganò. Automated Security Protocol Analysis with the AVISPA Tool. In Proceedings of the XXI Mathematical Foundations of Programming Semantics (MFPS'05). Electronic Notes in Theoretical Computer Science, to appear. 